\title{
Biodiversity and Ecosystem Functioning: Current Knowledge and Future Challenges
}

\author{
M. Loreau, ${ }^{1 *}$ S. Naeem, ${ }^{2}$ P. Inchausti, ${ }^{1}$ J. Bengtsson, ${ }^{3}$ J. P. Grime, ${ }^{4}$ A. Hector, ${ }^{5}$ D. U. Hooper, ${ }^{6}$ M. A. Huston, ${ }^{7}$ D. Raffaelli, ${ }^{8}$ \\ B. Schmid, 9 D. Tilman, $^{10}$ D. A. Wardle ${ }^{4}$
}

The ecological consequences of biodiversity loss have aroused considerable interest and controversy during the past decade. Major advances have been made in describing the relationship between species diversity and ecosystem processes, in identifying functionally important species, and in revealing underlying mechanisms. There is, however, uncertainty as to how results obtained in recent experiments scale up to landscape and regional levels and generalize across ecosystem types and processes. Larger numbers of species are probably needed to reduce temporal variability in ecosystem processes in changing environments. A major future challenge is to determine how biodiversity dynamics, ecosystem processes, and abiotic factors interact.

$\mathbf{T}$ he relationship between biodiversity and ecosystem functioning has emerged as a central issue in ecological and environmental sciences during the last decade. Increasing domination of ecosystems by humans is steadily transforming them into depauperate systems $(1,2)$. Because ecosystems collectively determine the biogeochemical processes that regulate the Earth system, the potential ecological consequences of biodiversity loss have aroused considerable interest (3-9).

Recent experimental and theoretical work in this area has also led to animated debates and controversies (10-14). Human impacts on the environment from local to global scales cause not only a general decline in diversity, but also predictable functional shifts as sets of species with particular traits are replaced by other sets with different traits $(15,16)$. This has resulted in

'Laboratoire d'Ecologie, UMR 7625, Ecole Normale Supérieure, 46 rue d'Ulm, F-75230 Paris Cedex 05, France. ${ }^{2}$ Department of Zoology, University of Washington, 24 Kincaid Hall, Box 351800, Seattle, WA 98195-1800, USA. ${ }^{3}$ Department of Ecology and Crop Production Science, Swedish University of Agricultural Sciences, Box 7043, SE-750 07 Uppsala, Sweden. ${ }^{4}$ Department of Animal and Plant Sciences, University of Sheffield, Sheffield, S10 2TN, UK. ${ }^{5}$ NERC Centre for Population Biology, Imperial College at Silwood Park, Ascot, Berks, SL5 7PY, UK. ' $D$ epartment of Biology, Western Washington University, 516 High Street, Bellingham, WA 98225-9160, USA. ${ }^{7}$ Environmental Sciences Division, Oak Ridge National Laboratory, Oak Ridge, TN 37831-6400, USA. ${ }^{8}$ Environment Department, University of York, York, YO10 5DD, UK. 9Institut für Umweltwissenschaften, Universität Zürich, Winterthurerstrasse 190, CH-8057 Zürich, Switzerland. ${ }^{10}$ Department of Ecology, Evolution and Behavior, University of Minnesota, St. Paul, MN 55108, USA.

*To whom correspondence should be addressed. Email: Loreau@ens.fr the current debate in which scientists disagree about the relative importance of functional substitutions and declining species richness as determinants of changes in ecosystem functioning. Comparative studies have begun to reveal the extent to which functional substitutions alter ecosystem properties such as productivity, decomposition rates, nutrient cycling, and resistance and resilience to perturbations $(17,18)$. On the other hand, a new wave of experimental studies has manipulated species richness by using synthesized model ecosystems in both terrestrial and aquatic environments (19-25). Both approaches suggest that a large pool of species is required to sustain the assembly and functioning of ecosystems in landscapes subject to increasingly intensive land use. It is not yet clear, however, whether this dependence on diversity arises from the need for recruitment of a few key species from within the regional species pool or is due to the need for a rich assortment of complementary species within particular ecosystems.

In this article, we seek to set a common framework to understand these issues, to move beyond past differences of opinion, and to define new perspectives, after a recent conference held in Paris. We do not attempt to comprehensively review these issues, elements of which can be found elsewhere (39). Rather, we focus on major regions where recent advances have been made.

\section{Experimentally Altered Diversity}

Although the first study that experimentally manipulated diversity did so across several trophic levels (19), later studies focused mainly on effects of plant taxonomic diversity and plant functional-group diversity on primary production in grassland ecosystems (20-23). Because plants, as primary producers, represent the basal component of most ecosystems, they represented the logical place to begin detailed studies. Several, although not all, experiments using randomly assembled communities found that primary production exhibits a positive relationship with plant species and functional-group diversity (Fig. 1).

These results attracted a great deal of interest, not only because they were novel, but also because they seemed counter to patterns often observed in nature, where the most productive ecosystems are typically characterized by low species diversity (26, 27). The controversy over the interpretation of these results started with the realization that they can be generated by different mechanisms. The mechanisms discussed so far may be grouped into two main classes. First are local deterministic processes, such as niche differentiation and facilitation, which increase the performance of communities above that expected from the performance of individual species grown alone. We will subsume them here under the term "complementarity" for convenience's sake. Second are local and regional stochastic processes involved in community assembly, which are mimicked in experiments by random sampling from a species pool. Random sampling coupled with local dominance of highly productive species can also lead to increased average primary production with increasing diversity, because plots that include many species have a higher probability of containing highly productive species $(10,11,28)$. Two issues are involved in this controversy: Are stochastic community assembly processes relevant? And what is the relative importance of the two classes of mechanisms?

There are diverging views on the relevance of the sampling component of biodiversity effects. As sampling processes were not an explicit part of the initial hypotheses, they have been viewed by some as "hidden treatments" (10), whereas others have viewed them as the simplest possible mechanism linking diversity and ecosystem functioning (28). This debate should be resolved through increasing knowledge about the patterns and processes of biodiversity loss in nature, which are still poorly known overall. If dom- 


\section{SCIENCE'S COMPASS}

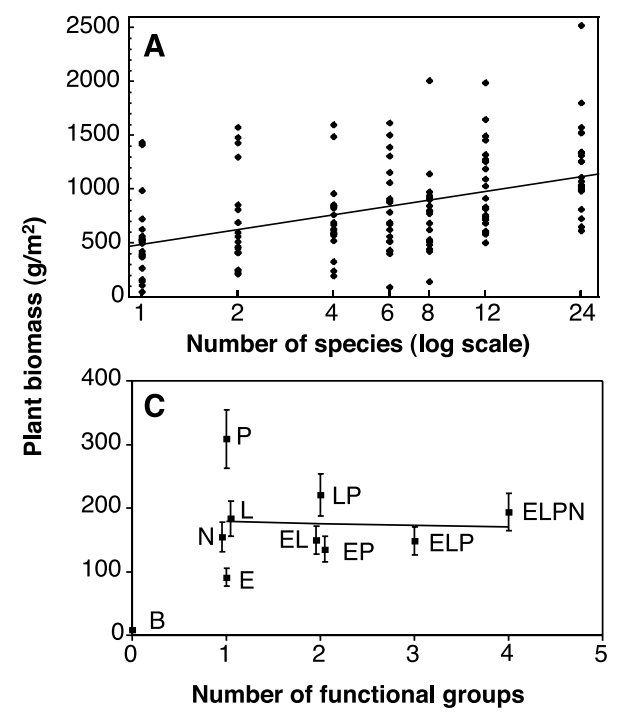

Fig. 1. Responses of total (A) or aboveground (B and $\mathbf{C}$ ) plant biomass (in grams per meter squared) to experimental manipulations of plant species richness (A and $B$ ) or functional-group richness (C) in grasslands in Minnesota (A) (31), across Europe (B) (23), and in California (C) (22). Points in (A) and (B) are data for individual plots. In (B) different regression slopes are shown for the eight sites to focus on betweenlocation differences rather then the general log-linear relationship re-

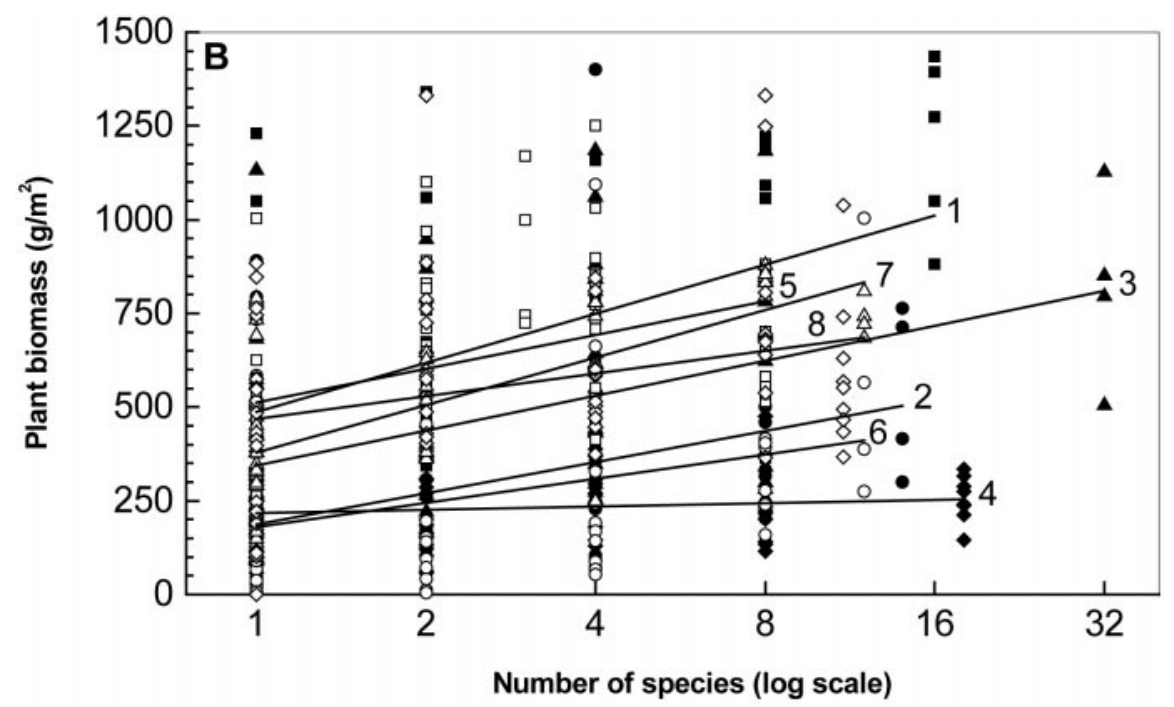

ported elsewhere (23). Filled squares and line 1, Germany; filled circles and line 2, Portugal; filled triangles and line 3, Switzerland; solid diamonds and line 4, Greece; open squares and line 5, Ireland; open circles and line 6. Sweden; open diamonds and line 7, Sheffield (UK); open diamonds and line 8, Silwood Park (UK). Symbols in (C) correspond to functional groups and their combinations: B, bare ground; E, early-season annuals; L, late-season annuals; P, perennial bunchgrasses; $N, N$ fixers. inant species control ecosystem processes and mostly rare species go extinct, the vagaries of community assembly or disassembly may have little relevance. But environmental changes and landscape fragmentation could prevent recruitment of appropriate dominants (29). Also, climate change could lead to gradual losses of species as abiotic conditions begin to exceed species' tolerance limits. Such losses could be random with respect to species effects on any given ecosystem process, leading to patterns of process response to changes in diversity similar to those observed in randomly assembled communities. It should be emphasized that recent experiments were not intended to reproduce any particular sequence of species loss; they reflect potential patterns, unaffected by correlations between diversity loss and compositional changes, rather than actual predictions of functional consequences of biodiversity loss under specific global change scenarios.

Recent experiments were also not designed to investigate detailed underlying mechanisms. Assessing the relative importance of complementarity and sampling effects has been done so far indirectly, by using comparisons between the performances of mixtures and monocultures $(14,23,30,31)$. Furthermore, it is becoming clear that complementarity and sampling are not mutually exclusive mechanisms as previously thought. Communities with more species have a greater probability of containing a higher phenotypic trait diversity. Dominance that is brought about by ecological "selection" of species with particular traits and complementarity among species with differ-

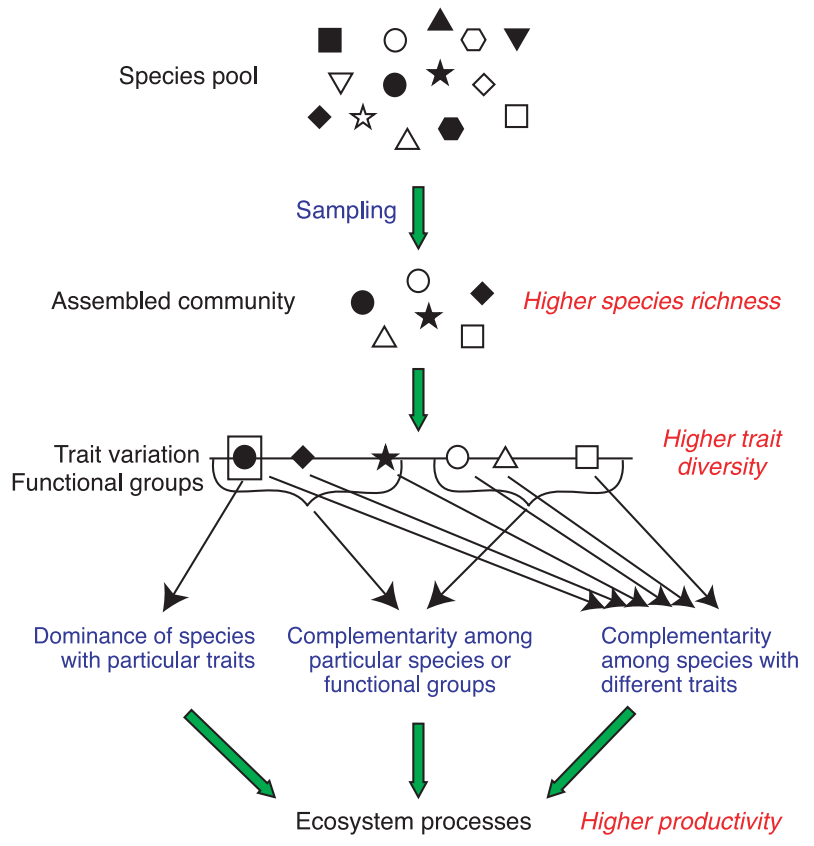

Fig. 2. Hypothesized mechanisms involved in biodiversity experiments using synthetic communities. Sampling effects are involved in community assembly, such that communities that have more species have a greater probability of containing a higher phenotypic trait diversity. Phenotypic diversity then maps onto ecosystem processes through two main mechanisms: dominance of species with particular traits, and complementarity among species with different traits. Intermediate scenarios involve complementarity among particular species or functional groups or, equivalently, dominance of particular subsets of complementary species.

ent traits are two ways by which this phenotypic diversity maps onto ecosystem processes (6). These two mechanisms, however, may be viewed as two poles on a continuum from pure dominance to pure complementarity. Intermediate scenarios involve complementarity among particular sets of species or functional groups, or dominance of particular subsets of complementary species (Fig. 2). Any bias in community assembly that leads to correlations between diversity and community composition may involve both dominance and complementarity.

Rigorously testing the hypothesis that there is a minimum subset of complementary species that is sufficient to explain diversity effects will often be difficult because it would ideally require testing, with replication, the performance of all species combinations at all diversity levels. Re-analysis of data from previously published experiments suggests significant effects of species richness on plant biomass even after controlling for the strong effects of certain species, such as legumes $(30,31)$. Although these new results presented at the Paris conference will need to be critically evaluated, they suggest that complementarity does occur among at least several 


\section{SCIENCE'S COMPASS}

species belonging to different functional groups in these experiments. No clear evidence, however, has been provided so far for complementarity among a large number of species, although complementarity among rare species would be difficult to detect. With our knowledge now, we cannot reject the hypothesis that a few dominant species suffice to provide the functional diversity that is necessary to explain the level of primary production observed in grassland ecosystems at the small spatial and temporal scales considered in recent experiments.

Future experiments should strive to overcome the limitations that led to the recent controversy. Greater attention should be paid to what individual species do in these experiments. One option for assembly experiments is to have carefully balanced designs to allow contrasts between plots with and without particular species or subsets of species. Another option is to include manipulations of evenness within a level of species richness, which could provide an alternative to methods based
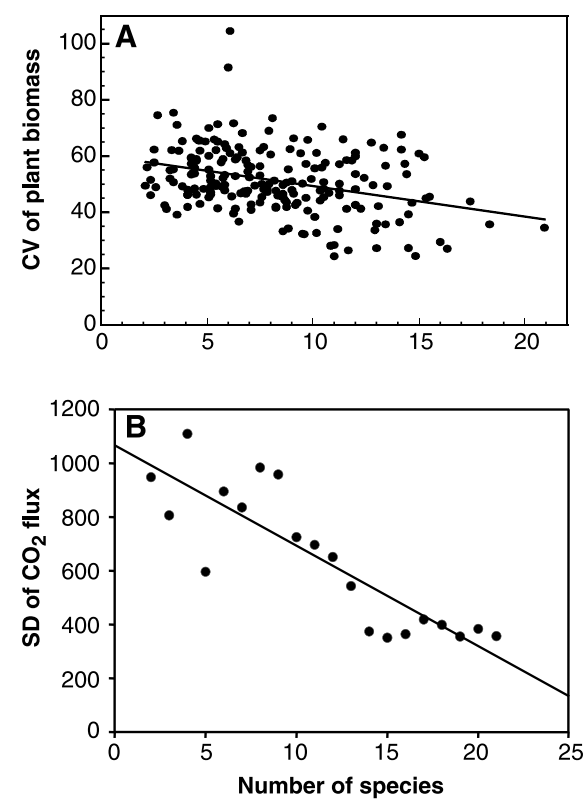

Fig. 3. Observed decreases in variability of ecosystem processes as species richness increases. Interpretation of these patterns, however, is complicated by the correlation of additional factors with species richness. (A) Adjusted coefficient of temporal variation of annual total plant biomass (in grams per meter squared) over 11 years for plots differing in number of species in experimental and natural grasslands in Minnesota (38). The correlation of variations in soil nitrogen with species richness in these plots precludes the interpretation of increased stability as a pure diversity effect (10), although the diversity effect remained significant even after controlling for potentially confounding variables (36). (B) Standard deviation of $\mathrm{CO}_{2}$ flux (in microliters per 18 hours) from microbial microcosms (24). In these data, temporal variability in response to diversity is confounded with between-replicate variability. on comparisons with monocultures, to separate dominance and complementarity effects. There is also a great need for other approaches based on "natural" ecosystems, such as removal experiments (32) and comparative approaches that control for variation of factors other than diversity (33).

\section{Biodiversity as Insurance}

Even when high diversity is not critical for maintaining ecosystem processes under constant or benign environmental conditions, it might nevertheless be important for maintaining them under changing conditions. The insurance hypothesis (34) and related hypotheses (35-40) propose that biodiversity provides an "insurance" or a buffer, against environmental fluctuations, because different species respond differently to these fluctuations, leading to more predictable aggregate community or ecosystem properties. In this hypothesis, species that are functionally redundant for an ecosystem process at a given time are no longer redundant through time.

In a way, this is the old stability-versuscomplexity debate resurfacing in a new form (7). Several problems, however, have confused this historical controversy: (i) The general concept of "stability" actually covers a wide array of different properties (41); (ii) the relationship between these properties and diversity may change across ecological levels of organization such that large variability at the population level may not imply large variability of ecosystem processes $(38,41)$; and (iii) stability has been approached mainly within a deterministic, equilibrium theoretical framework. Recent theoretical work has attempted to remove these obstacles and has provided support for the insurance hypothesis. As diversity increases, the variability of individual populations may increase as a result of the destabilizing influence of strong species interactions internal to the system, but the variability of aggregate ecosystem properties often decreases because of the stabilizing influence of asynchronous spe- cies responses to intrinsic or extrinsic environmental fluctuations (34-40). What remains unclear, however, is whether this stabilizing effect saturates at low or high diversity, which depends on model conditions $(5,9,34,38,39)$.

Whereas experimental work has played a leading role regarding short-term effects of biodiversity on ecosystem functioning, theory has been prominent in the diversitystability debate, both historically and recently. A number of empirical and experimental studies have shown decreased variability of ecosystem processes as diversity increases (Fig. 3). These studies, however, have been based either on diversity gradients established naturally or after other treatments $(36,38)$, or on microcosm experiments in which variability among replicates was also considered $(24,25)$, which does not fully preclude alternative interpretations $(10,13)$. Experiments in which both diversity and environmental fluctuations are controlled are now needed to perform rigorous tests of the insurance hypothesis.

Theory too should evolve to provide better guidance for experiments. Most of the classical equilibrium approaches may be inadequate to understand stability properties such as resilience and resistance at the ecosystem level. New approaches should be developed that take into account the dynamics of diversity and the potential for adaptation through phenotypic plasticity, evolutionary changes, and species replacement.

\section{From Experiments to Patterns}

The relationship between productivity and diversity has long been studied from an angle different from that in recent experimental studies. It is often, although not always, described by a hump-shaped curve, in which diversity is considered a function of productivity (Fig. 4A) $(26,27)$. These curves have typically been obtained by using correlations across different sites or nutrient addition treatments. Some comparative approaches have also suggested neg-
Fig. 4. Hypothesized relationships between (A) diversityproductivity patterns driven by environmental conditions across sites, and (B) the local effect of species diversity on productivity. (A) Comparative data often indicate a unimodal relationship between diversity and productivity driven by changes in environmental conditions. (B) Experimental variation in species richness under a specific set of environmental conditions

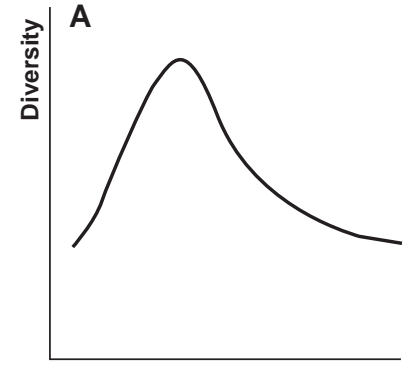

Productivity Soil and climate effects

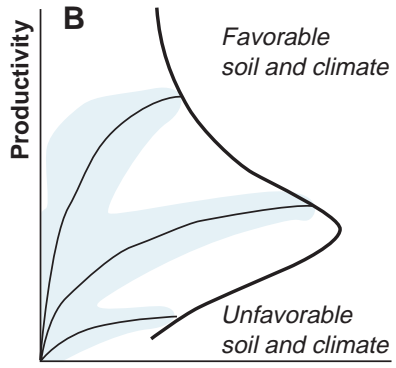

Diversity produces a pattern of decreasing between-replicate variance and increasing mean response with increasing diversity, as indicated by the thin, curved regression lines through the scatter of response values (shaded areas). 


\section{SCIENCE'S COMPASS}

ative relationships between plant species evenness and rates of various ecosystem processes (42). The differences between these large-scale, observational approaches and the small-scale, experimental approaches have also generated debate (12). How can these results be reconciled?

The two approaches examine different causal relationships under different sets of conditions. The classical approach attempts to identify the causes of spatial variation in diversity across environmental gradients. Variation in diversity is often correlated with productivity, but also with many other factors that influence productivity, such as soil fertility, climate, disturbance regime, or herbivory. The recent experimental approach examines whether diversity alone has a local effect on productivity within each site, when all these other factors are held constant. The two approaches can be reconciled by considering that spatial patterns reveal correlations between diversity and productivity driven by environmental factors, whereas small-scale experiments reveal the effects of species properties and diversity on productivity that are detected after the effects of other environmental factors have been removed (Fig. 4B) (6).

Whether biodiversity loss will affect large-scale patterns of productivity hinges on the shape and steepness of the local dependence of productivity on diversity. Generally speaking, the relative effects of individual species and species richness may be expected to be greatest at small-to-intermediate spatial scales, but these biological factors should be less important as predictors of ecosystem processes at regional scales, where environmental heterogeneity is greater. Whereas diversity was manipulated as the independent variable in recent experiments, at large scales species diversity itself is a dynamical variable and adjusts to changes in environmental conditions. Abiotic factors then tend to be the main drivers of variations in ecosystem processes across environmental gradients (43).

Diversity loss at regional scales and dispersal limitations due to landscape fragmentation, however, will very likely feed back and reduce the pool of potential colonists at local scales and hence the potential for local compositional adjustments to environmental changes. Species-area relations imply that the long-term maintenance of a given level of diversity at local scales requires a much higher diversity at regional scales (44). One of the most potent effects of declining diversity could be the decline in the rate at which appropriate potential dominants are recruited during ecosystem assembly (29).

To understand and predict changes in biodiversity and ecosystem processes at large scales, therefore, we need to move beyond unidirectional causality approaches in which diversity is either cause or effect, and address feedbacks among biodiversity changes, ecosystem functioning, and environmental factors. Relationships between local, landscape, and regional scales also require particular attention.

\section{Generalizing Across Ecosystems}

Most of the recent experiments that found significant effects of species diversity have concerned effects of plant diversity on primary production and nutrient retention in temperate grasslands, both of which are under direct plant control. These and other experiments have often failed to detect significant effects on below-ground decomposition processes $(19,45)$, perhaps because these processes are under microbial control. This questions whether results obtained on primary production in grasslands can be generalized to other processes and ecosystems.

Plants can affect soil processes either directly, by stimulating or inhibiting decomposition rates, or indirectly, through increased primary production, by enhancing decomposition fluxes. Although some experiments found positive effects of plant diversity on soil microbial processes (46), experiments using litter addition, cotton strips, or litter mixing often showed variable and weak effects of plant diversity on decomposition rates (45). Current evidence suggests that properties of individual plant species are more important than plant diversity in governing soil process rates. This conclusion is echoed by theoretical work predicting that plant chemical quality diversity should decrease or not affect long-term nutrient recycling efficiency and productivity (47). In contrast, increased primary production generated by higher plant diversity is expected to stimulate secondary productivity. More generally, diversity changes at one trophic level may lead to a variety of potential responses for processes at higher trophic levels (48).

Species diversity in consumer trophic levels can also have complex effects on production at these and lower levels. Complementarity and sampling effects should tend to improve resource exploitation just as in plants. This should lead to higher secondary productivity if bottomup control prevails, as in plant-decomposer interactions (47). Enhanced resource exploitation, however, can lead to overexploitation, and thus decreased productivity, if top-down control is important, as might be the case with herbivores and predators. There have been few experiments to test these hypotheses. Recent microcosm experiments found significant effects of bacterial diversity on bacterial and algal biomasses (49) and of diversity of leaf-eating insects on decomposition rates (50), but others suggested that individual species and functional composition were the most important factors (51-53). The functional role of diversity in mutualistic interactions has also been poorly studied despite their importance in the maintenance of ecosystem processes, as shown by one experiment on mycorrhizal fungal diversity (54). Although there is a clear case for incorporating multiple trophic levels into studies of biodiversity-ecosystem functioning relationships, logistical constraints, such as the high mobility of herbivores and carnivores and the difficulty of taxonomic identification of decomposers, partly explains why so few studies have done so as yet. Of particular importance are the vast areas of biodiversity that involve small organisms such as viruses, bacteria, archaea, protists, and microarthropods, which drive the bulk of ecosystem processes. For example, chemical transformations in the nitrogen cycle are predominantly driven by prokaryotic organisms, as is decomposition of organic matter. Modern molecular tools are beginning to make possible the integration of microbial diversity into studies of ecosystem processes.

There is also a need to extend our current knowledge to ecosystem types other than temperate grasslands, such as forest, freshwater, and marine (55) ecosystems. Top-down control is often thought to be more common in freshwater than in terrestrial ecosystems (56); significant differences might then be expected between ecosystem types just as between trophic levels. Generally speaking, differences in coexistence mechanisms may lead to differences in biodiversity effects on ecosystem functioning. For example, in disturbance-driven systems, the colonization ability and growth rate of individual species, rather than niche complementarity, might drive ecosystem processes.

\section{Conclusions}

Significant advances in science occur when observational, experimental, and theoretical studies coincide. Recent work has done this to some extent for studies of the effects of diversity on productivity and temporal stability at local scales, although much additional work is still needed, in particular to apply results to larger spatial scales. There is consensus that at least some minimum number of species is essential for ecosystem functioning under constant conditions and that a larger number of species is probably essential for maintaining the stability of ecosystem processes in changing environments. Determining which species have a significant impact on which processes in which ecosystems, however, remains an open empirical question.

There are many reasons - including aesthetic, cultural, and economic - why we may wish to conserve biodiversity. From a strictly functional point of view, species matter so far as their individual traits and interactions contribute to maintain the functioning and stability of ecosystems and biogeochemical cycles. Although species richness is easier to mea- 


\section{SCIENCE'S COMPASS}

sure, a more predictive science might be achieved if appropriate functional classifications were devised $(26,27,57,58)$. Specific knowledge of functional types may be critical to predict ecosystem responses under different global change scenarios, or where management priorities seek to manipulate species composition directly, for example, in complex agroecosystems, forestry, or ecosystem restoration with particular functional goals in mind.

The traditional approach in community ecology has considered species diversity as a dependent variable controlled by abiotic conditions and ecosystem-level constraints. The traditional approach in ecosystem ecology has primarily focused on dominant species as biotic controllers of ecosystem processes. Recent approaches have broadened the perspectives of both subdisciplines by assessing the role of biodiversity as a potential modulator of processes. In reality, there are mutual interactions among biodiversity changes, ecosystem functioning, and abiotic factors. Integrating these interactions into a single, unified picture, both theoretically and experimentally, and across ecosystem types and processes, is a major challenge which may help bring about a true synthesis of community and ecosystem ecology.

\section{References and Notes}

1. P. M. Vitousek, H. A. Mooney, J. Lubchenco, J. M. Melillo, Science 277, 494 (1997).

2. O. E. Sala et al., Science 287, 1770 (2000).

3. Biodiversity and Ecosystem Function, E.-D. Schulze, H. A. Mooney, Eds. (Springer Verlag, Berlin, 1993).
4. F. Schläpfer, B. Schmid, Ecol. Appl. 9, 893 (1999).

5. M. W. Schwartz et al., Oecologia 122, 297 (2000).

6. M. Loreau, Oikos 91, 3 (2000).

7. K. S. McCann, Nature 405, 228 (2000)

8. F. S. Chapin III et al., Nature 405, 234 (2000).

9. K. L. Cottingham, B. L. Brown, J. T. Lennon, Ecol. Lett. 4, 72 (2001)

10. M. A. Huston, Oecologia 110, 449 (1997).

11. L. W. Aarssen, D. Tilman, Oikos 80, 183 (1997)

12. D. Tilman et al., Science 278, 1865 (1997)

13. D. A. Wardle, S. Naeem, S. Li, Nature 394, 30 (1998)

14. M. A. Huston et al., Science 289, 1255a (2000).

15. D. McCollin, L. Moore, T. Sparks, Biol. Conserv. 92, 249 (2000).

16. J. P. Grime et al., Science 289, 762 (2000).

17. C. W. MacGillivray et al., Funct. Ecol. 9, 640 (1995).

18. J. P. Grime et al., Oikos 79, 259 (1997).

19. S. Naeem, L. J. Thompson, S. P. Lawler, J. H. Lawton, R. M. Woodfin, Nature 368, 734 (1994).

20. D. Tilman, D. Wedin, J. Knops, Nature 379, 718 (1996).

21. D. Tilman et al., Science 277, 1300 (1997)

22. D. U. Hooper, P. M. Vitousek, Science 277, 1302 (1997).

23. A. Hector et al., Science 286, 1123 (1999).

24. J. McGrady-Steed, P. M. Harris, P. J. Morin, Nature 390, 162 (1997).

25. S. Naeem, S. Li, Nature 390, 507 (1997).

26. M. A. Huston, Biological Diversity (Cambridge Univ. Press, Cambridge, 1994).

27. J. P. Grime, Plant Strategies, Vegetation Processes and Ecosystem Properties (Wiley, New York, 2nd ed., 2001).

28. D. Tilman, C. Lehman, K. Thompson, Proc. Natl. Acad. Sci. U.S.A. 94, 1857 (1997)

29. J. P. Grime, J. Ecol. 86, 902 (1998).

30. M. Loreau, A. Hector, Nature 412, 72.

31. D. Tilman, in Ecology: Achievement and Challenge, M. C. Press, N. J. Huntly, S. A. Levin, Eds. (British Ecological Society Symp. Vol. Ser. 41, Blackwell Science, Oxford, 2001), pp. 183-207.

32. D. A. Wardle et al., Ecol. Monogr. 69, 535 (1999).

33. A. Y. Troumbis, D. Memtsas, Oecologia 125, 101 (2000).

34. S. Yachi, M. Loreau, Proc. Natl. Acad. Sci. U.S.A. 96, 1463 (1999)
35. S. J. McNaughton, Am. Nat. 111, 515 (1977).

36. D. Tilman, Ecology 77, 350 (1996).

37. D. F. Doak et al., Am. Nat. 151, 264 (1998).

38. D. Tilman, Ecology 80, 1455 (1999).

39. C. L. Lehman, D. Tilman, Am. Nat. 156, 534 (2000).

40. A. R. Ives, J. L. Klug, K. Gross, Ecol. Lett. 3, 399 (2000).

41. S. L. Pimm, Nature 307, 321 (1984).

42. D. A. Wardle, O. Zackrisson, G. Hörnberg, C. Gallet Science 277, 1296 (1997).

43. M. Loreau, Proc. Natl. Acad. Sci. U.S.A. 95, 5632 (1998).

44. D. Tilman, Science 286, 1099 (1999)

45. D. A. Wardle, K. I. Bonner, K. S. Nicholson, Oikos 79 247 (1997)

46. A. Stephan, A. H. Meyer, B. Schmid, J. Ecol. 88, 988 (2000).

47. M. Loreau, Proc. R. Soc. London Ser. B 268, 303 (2001).

48. N. M. Haddad, D. Tilman, J. Haarstad, M. Ritchie, J. M. H. Knops, Am. Nat. 158, 17 (2001).

49. S. Naeem, D. R. Hahn, G. Schuurman, Nature 403 $762(2000)$

50. M. Jonsson, B. Malmqvist, Oikos 89, 519 (2000).

51. J. Mikola, H. Setälä, Oikos 83, 180 (1998)

52. J. Laakso, H. Setälä, Oikos 87, 57 (1999).

53. J. Norberg, Oecologia 122: 264 (2000).

54. M. van der Heijden et al., Nature 396, 69 (1998).

55. M. C. Emmerson, M. Solan, D. M. Paterson, D. Raffaelli, Nature 411, 73 (2001).

56. D. R. Strong, Ecology 73, 747 (1992)

57. S. Lavorel, S. Mclntyre, J. Landsberg, T. D. A. Forbes, Trends Ecol. Evol. 12, 474 (1997).

58. F. D. Hulot, G. Lacroix, F. Lescher-Moutoué, M. Loreau, Nature 405, 340 (2000)

59. The conference "Biodiversity and ecosystem functioning: Synthesis and perspectives" was held from 6 to 9 December 2000 as a contribution to the International Geosphere-Biosphere Programme-Global Change and Terrestrial Ecosystems (IGBP-GCTE) Focus 4 and Diversitas Core Programme Element 1. We gratefully acknowledge financial support by the European Science Foundation, the Centre National de la Recherche Scientifique (France), and the U.S. National Science Foundation. We thank all other conference participants for stimulating discussions.

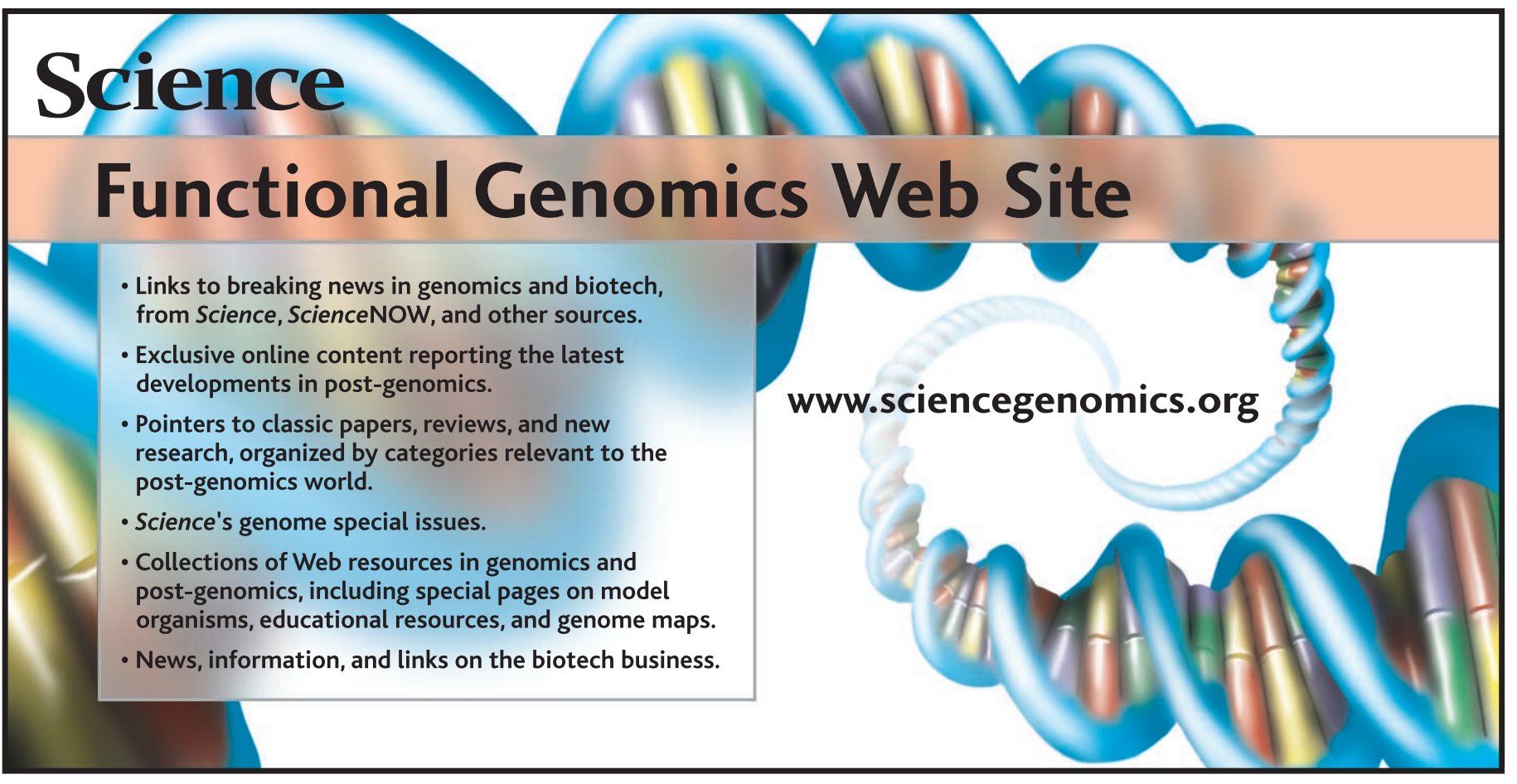

